\title{
Self-maintenance of intoxication in the rat: A modified replication'
}

\author{
R. J. SENTER AND J. D. SINCLAIR \\ UNIVERSITY OF CINCINNATI
}

Variable interval schedules of food reinforcement were found to produce excessive drinking of both EtOH solution and Sucaryl. The polydipsia during training did not increase subsequent preferences for the solutions. It is concluded that excessive drinking of alcohol associated with such treatment is not analogous to alcoholic habituation.

Falk (1961) reported that rats pressing a bar for food reinforcement on a variable interval schedule consumed three times as much water in $3 \mathrm{~h}$ as they normally drank in $24 \mathrm{~h}$. Lester (1961) demonstrated that if an alcohol solution were substituted for water, Falk's procedure could cause rats to consume enough alcohol to keep themselves in a state of intoxication.

The present experiment is a replication of Lester's study with additional controls. The hypothesis that alcohol addiction might possibly result from such treatment was tested by making subsequent measures of preference and comparing these measures with preference measures obtained from untreated animals. Method

Twenty-two $250 \mathrm{~g}$ male hooded rats were given limited access to Rockland Mouse/Rat Diet until they were reduced to approximately 200 g. Each rat was placed in a Skinner box on four consecutive days for $3 \mathrm{~h}$ training sessions. Shaping was accomplished by placing six 45-mg food pellets in the food cup and smashing two additional pellets on the Plexiglas bar. Each of the first 100 presses produced a $45 \mathrm{mg}$ pellet in the food cup. For the next 100 reinforcements, pellets were given only for bar presses during $2 \mathrm{sec}$ intervals separated by nonreinforcement periods averaging $19 \mathrm{sec}$ (range 5 to $38 \mathrm{sec}$ ). The nonreinforcement periods were then lengthened to a mean of 30 sec (range 7 to $60 \mathrm{sec}$ ) for the third set of 100 reinforcements, and eventually to $55 \mathrm{sec}$ (range 10 to $110 \mathrm{sec}$ ) for the remainder of the training time.

During the training sessions, 12 rats (Group $\mathrm{TA}$ ) had ad lib access to a $5.6 \%$ (V/V) EtOH solution from Richter bottles. The other 10 rats (Group TS) received, in place of the alcohol solution, a Sucaryl ${ }^{2}$ solution (1 compound sodium cyclamate tablet per $100 \mathrm{ml}$ of water). Following each $3 \mathrm{~h}$ session all animals were returned to their home cages where there was ad lib access to water.

Food, water, and the solution used in training were continually available on an ad lib basis in the home cages the three weeks following the fourth training session. Records were taken of the water and "training" solution drunk daily during this post-training period.
These data were then converted into measures of preference by dividing the volume of solution consumed, either EtOH or Sucaryl, by the total volume of fluid consumed daily. The preference measures are referred to as $E / T$ for EtOH and $S / T$ for Sucaryl.

Two additional control groups of rats that did not receive the training sessions were used. Group UA, consisting of eight untrained rats, received ad lib access to the alcohol solution, and Group US, consisting of seven rats, received access to the Sucaryl solution. Both groups had food and water available at all times. All animals were male hooded rats. The animals in Group US had been used in an earlier experiment in which they had consumed some alcohol, but they had received no experience with the Sucaryl solution on which they were subsequently tested. Resulis

Lester's (1961) findings were replicated. The TA rats drank progressively greater amounts of alcohol under longer periods of nonreinforcement. Most of the rats showed signs of being intoxicated by the end of the training sessions, e.g., inability to stand on the hind legs, and inability to keep the feet from slipping through the bars of the cage. The group receiving Sucaryl (TS) drank even more fluid than the TA animals did ( $F_{T S}$ vs $T A=26.3, d f=1 / 230$, $p<.001)$. The amount consumed by the TS rats was almost exactly twice as much as that consumed by the TA rats at all stages of the treatment, as can be seen in Fig. 1. However, data from Falk's (1961) experiment, which can be compared to Day 4 of the present study, indicated that more pure water than Sucaryl solution is consumed under such treatment.

There was a significant increase in the consumption of both alcohol and Sucaryl solutions across days of training $\left(F_{\text {Days }}=31.8, d f=3 / 60, p<.001\right)$, but there was more absolute increase in the consumption of Sucaryl solution ( $F_{T S}$ vs TA by Days $=5.71$, df $=3 / 60$, $\mathrm{p}<.01$ ).

Training had little effect on the posttraining preference for either alcohol or Sucaryl solution $\left(F_{T}\right.$ vs $U=$ 1.36, $\mathrm{df}=1 / 32, \mathrm{p}>.05$ ). As can be seen in Fig. 2, the tendency was for training to lower the preference for both solutions; Groups TS and TA generally showed lower preference than did Groups US and UA for Sucaryl and alcohol, respectively. Surprisingly, the relatively higher consumption of Sucaryl observed during the training period disappeared in the posttraining period ( $F_{T S}$ vs $T A=.03$ ). The absolute consumption of alcohol subsequent to training did not 
differ appreciably from that of the untrained animals. Group TA had a mean intake of $13.86 \mathrm{ml}$ of EtOH/rat/ day, while Group UA had a mean intake of $14.91 \mathrm{ml}$ of $\mathrm{EtOH} / \mathrm{rat} / \mathrm{day}$.

A significant increase in preference over the posttraining day was shown by all groups except US. The interaction of all four groups over time was also sigmificant ( $F=1.85$, df $=20 / 640, p<.05$ ).

Lester reported that a characteristic pattern of drinking appeared, 1.e., the rats usually drank immediately following food reinforcement. "The rats acted as if the bar pressing and the drinking were both necessary to obtain food," (Lester, 1961, p. 229). In the present experiment records were kept of each bar press, food reinforcement, and contact with the alcohol solution, in order to identify any pattern that might be present. The only patterns which appeared were idiosyncratic, and even these were not consistent over time. "Superstitious behavior" did occur: one rat which happened to receive a pellet while chewing

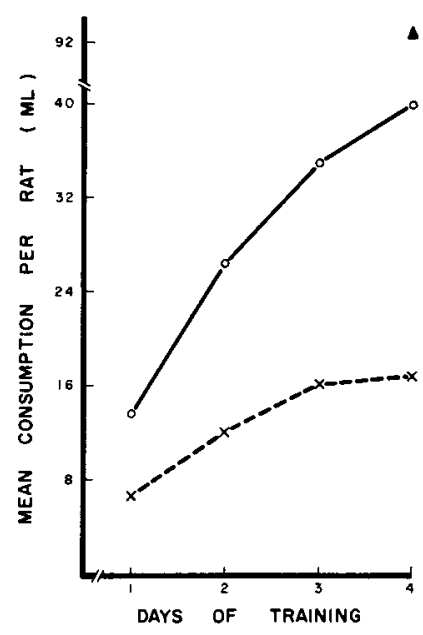

Fig. 1. Consumption of alcohol and Sucaryl solutions during training. Data for water are adapted from Falk (1961).

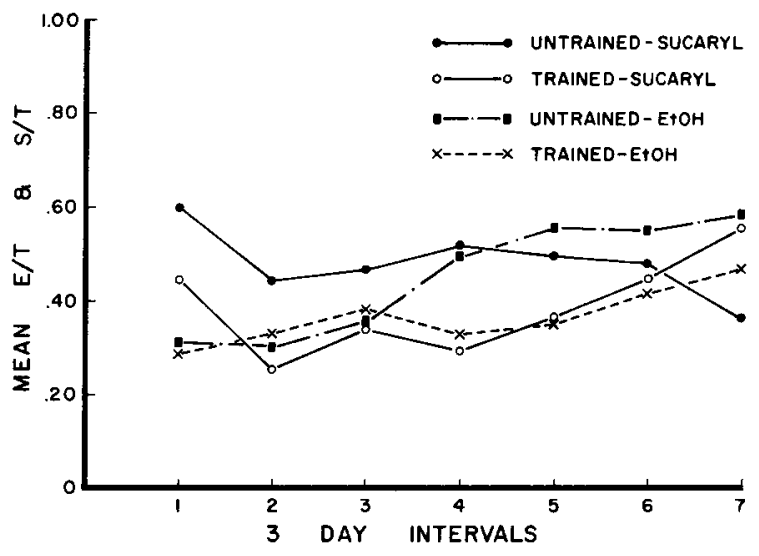

Fig. 2. Preference for alcohol and Sucaryl during the posttraining period by trained and untrained rats.

on the bar continued chewing on the Plexiglas quite vigorously throughout the training sessions. Although the drinking behavior was also vigorous, the lack of a consistent pattern suggests that the observed polydipsia is not a form of "superstitious behavior." Discussion

The present study has indicated that a variable interval schedule of food reinforcement can produce excessive drinking of an alcohol solution to the point of intoxication. Such voluntarily produced intoxication and excessive drinking does not increase the subsequent preference for alcohol. The extreme consistency of the phenomenon, however, suggests it as a useful tool in the study of alcoholism.

\section{References}

FALK, J. L. Production of polydipsia in normal rats by an intermittent food schedule. Science, 1961, 133, 195-196.

LESTER, D. Self-maintenance of intoxication in the rat. Quart. J. Stud. Alcohol, 1961, 22, 223-231.

Notes

1. This study represents a portion of the effort carried out under USPHS Grant No. MH 11895-01.

2. Available from Abbott Laboratories, North Chicago, Illinois. 